\title{
Depth Penetration and Scope Extension of Failures in the Cascading of Multilayer Networks
}

\author{
Wen-Jun Jiang, Run-Ran Liu $(\mathbb{D}$, and Chun-Xiao Jia 10 \\ Research Center for Complexity Sciences, Hangzhou Normal University, Hangzhou, Zhejiang 311121, China \\ Correspondence should be addressed to Run-Ran Liu; runranliu@163.com
}

Received 16 December 2019; Revised 7 March 2020; Accepted 17 March 2020; Published 25 April 2020

Guest Editor: Tomas Veloz

Copyright (c) 2020 Wen-Jun Jiang et al. This is an open access article distributed under the Creative Commons Attribution License, which permits unrestricted use, distribution, and reproduction in any medium, provided the original work is properly cited.

\begin{abstract}
Real-world complex systems always interact with each other, which causes these systems to collapse in an avalanche or cascading manner in the case of random failures or malicious attacks. The robustness of multilayer networks has attracted great interest, where the modeling and theoretical studies of which always rely on the concept of multilayer networks and percolation methods. A straightforward and tacit assumption is that the interdependence across network layers is strong, which means that a node will fail entirely with the removal of all links if one of its interdependent nodes in other network layers fails. However, this oversimplification cannot describe the general form of interactions across the network layers in a real-world multilayer system. In this paper, we reveal the nature of the avalanche disintegration of general multilayer networks with arbitrary interdependency strength across network layers. Specifically, we identify that the avalanche process of the whole system can essentially be decomposed into two microscopic cascading dynamics in terms of the propagation direction of the failures: depth penetration and scope extension. In the process of depth penetration, the failures propagate from layer to layer, where the greater the number of failed nodes is, the greater is the destructive power that will emerge in an interdependency group. In the process of scope extension, failures propagate with the removal of connections in each network layer. Under the synergy of the two processes, we find that the percolation transition of the system can be discontinuous or continuous with changes in the interdependency strength across network layers, which means that a sudden system-wide collapse can be avoided by controlling the interdependency strength across network layers. Our work not only reveals the microscopic mechanism of global collapse in multilayer infrastructure systems but also provides stimulating ideas on intervention programs and approaches for cascade failures.
\end{abstract}

\section{Introduction}

Many real-world complex systems, both natural [1] and man made [2-5], can be described as multilayer or interdependent networks given the existence of different levels of interdependence across network layers. Recent theoretical studies on networks with two or more layers show that when the nodes in each network are interdependent on the nodes in other networks, even small initial failures can propagate back and forth and lead to the abrupt collapse of the whole system [5-10]. In this sense, multilayer networks are more fragile than single-layer networks in resisting the propagation of initial failures [6]. In recent years, we have witnessed considerable progress in the study of multilayer networks with the aid of percolation theory [11-13]. It has been found that multilayer networks are not as fragile as in theoretical studies under certain specific conditions such as those given link overlap $[14,15]$, geometric correlations $[16,17]$, correlated community structures [18], interlayer degree correlations $[19,20]$, intralayer degree correlations [21], asymmetry in interdependence [22], and autonomous nodes [23-25] being able to facilitate the viability of nodes and alleviate the suddenness of the collapse in an interdependent system. In addition, some features of real interdependent systems, such as spatial constraints [26-30], clustering [31,32], and degree distribution [33, 34], also enhance the robustness and mitigate cascading failures of interdependent networks.

A key question in the modeling of multilayer networks is how to describe the interdependencies across network layers. A straightforward method employed in most previous models of cascading failures in multilayer networks is 
assuming that the layer interdependence is "strong," where a failure node can cause all of its interdependent neighbors to fail completely $[6,23,35-40]$. This assumption has already been extended extensively to the study of cascading dynamics in networks under different conditions such as interdependency groups in single-layer networks [41-43] and $\mathrm{k}$-core percolation [44], weak percolation [45], and redundant percolation [46] in multilayer networks. Nevertheless, this assumption is somewhat simplistic and cannot cover the case where nodes are weakly interdependent. For instance, in a civil transportation system, the flow of passengers from city to city depends on a number of transportation modes such as coaches, trains, airplanes, and ferries. When any mode becomes unavailable, the total failure of the other three modes seems impossible, e.g., when a local train station is shut down, passenger flow into the city may be decreased: some passengers destined for this city may cancel their trips, and the transferring passengers would switch to other cities to reach their destinations. The reduction of passenger flow can cause some routes in other modes to not operate properly, and carriers experience financial or other losses; for instance, airlines may cancel flights if passenger numbers are below expectations. Specifically, the interdependence across network layers can be "weak," and the failure of a node cannot destroy all of its dependency nodes in other network layers with certainty $[47,48]$. Under these circumstances, the failure of a train station can cause one or more of its interdependent nodes in other network layers to suffer damage or even failure, e.g., the failure of a local coach station, which can further lead to failures in more modes and deteriorate the connectivity of the city. By this token, there may exist a cascading process underlying a group of interdependent nodes across network layers, which means that the microscopic mechanism of global collapse in multilayer networks could include not only the propagation of failures from node to node inside a certain network layer but also the cascading process of failures across network layers. In this paper, we regard the propagation of failures inside a network layer as "inner-layer cascading" and the propagation of failures in a dependency group across network layers as "cross-layer cascading."

Previous networks that have considered the "strong" interdependence ignore the microscopic process of "crosslayer cascading," as the failure of one node will destroy all its interdependent neighbors. In this paper, we aim to model the cascading dynamics in multilayer networks within a more general situation by using the assumption of "weak" interdependence [47], where the strength of interdependence can be tuned by introducing a tolerance parameter $\alpha$. Using a comprehensive theoretical study and numerical simulations, we find that the cascading dynamic in multilayer networks is essentially the synergistic result of "crosslayer cascading" and "inner-layer cascading." In particular, we find that the system can undergo different types of percolation with changing tolerance parameters $\alpha$. Specifically, the system percolates as an abrupt (first-order) percolation transition for small values of $\alpha$. With increasing $\alpha$ exceeding a critical value $\alpha_{c}$, the system percolates in a continuous (second-order) manner. However, for scale-free networks, the phenomenon of double phase transitions occurs for some moderate parameter values of $\alpha$, where the networks in the system first percolate in a continuous (second-order) manner and then experience a first-order phase transition in an abrupt manner at another phase transition point.

\section{Model}

We consider a multilayer network consisting of $M$ layers of networks, where each network layer has $N$ nodes. We label the network layers with Latin letters $A, B, C$, and so on, and the nodes in each network layer are labeled with Arabic numbers $1,2, \ldots, N$. Therefore, each node in a certain network layer can be identified as a pair of coordinates $(x$, $X$ ), with $x$ denoting the node label and $X$ denoting the layer label. The nodes across network layers with the same Arabic number are interdependent on each other, i.e., they are replica nodes. As shown in Figure 1, the nodes $(1, A),(1, B)$, $(1, C)$, and $(1, D)$ are interdependent nodes in network layers $A, B, C$, and $D$, respectively. When one of these nodes fails, the other three nodes will suffer damages. Similarly, the other nodes can be also divided into several interdependent groups by their Arabic number labels. The nodes in the same network layer $X$ are linked by a set of connectivity links, and the connectivity degree of nodes follows a degree distribution $p_{k}^{X}$. In this paper, we consider the case where the $M$ network layers within the multilayer system have an identical degree distribution for simplicity.

The cascading in the multilayer networks is triggered by randomly selecting a fraction $1-p$ of nodes and their replicas as initially failed nodes. For each network layer, all the links of failed nodes are removed simultaneously, which breaks the network layer into a number of connected components [49]. The nodes in the isolated components are treated as failed ones, and the nodes in the giant component are functional. Due to the interdependency among the replica nodes across network layers, a failed node will cause a certain level of damage to it replicas, where the damage degree is controlled by the tolerance parameter $\alpha$. Specifically, when one node in a network layer fails, each connectivity link of its viable interdependency replicas in other network layers will be removed with a probability $1-\alpha$. Along with the removal of the connectivity links, the network layers will be further fragmented and, thus, lead to more failures. After a number of iterations of link removal caused by node failures and node isolations resulting from network fragmentation, the system can reach a steady state. In this iterative process, the failures can propagate from layer to layer through the interdependencies among replica nodes, and the failures can also propagate from node to node along with the removal of connectivity links in a certain network layer simultaneously. Here, we regard the propagation of failures inside a network layer as "scope extension" and the propagation of failures in an interdependency group across network layers as "depth penetration." Especially, it should be noticed that there is a microscopic cascading in the process of depth penetration: a failed node can lead more node failures in its replicas and, thus, lead more and more 


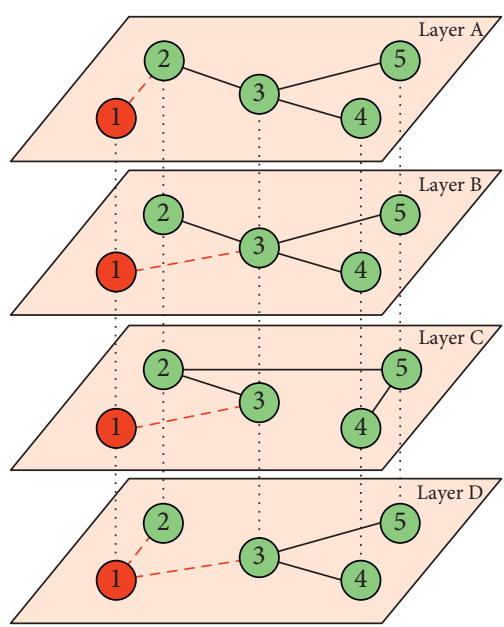

(a)

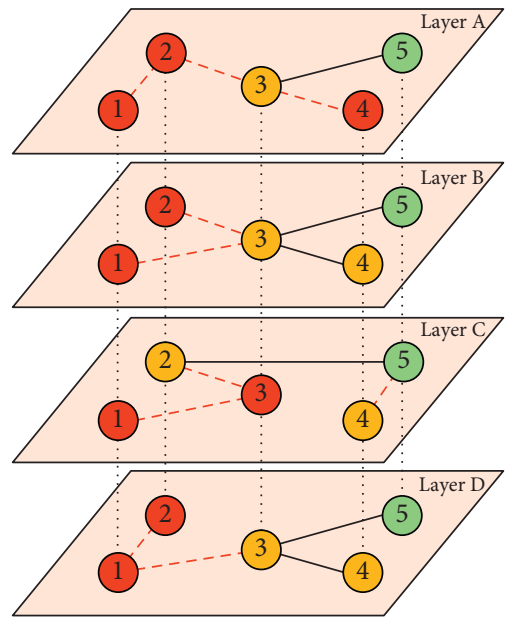

(d)

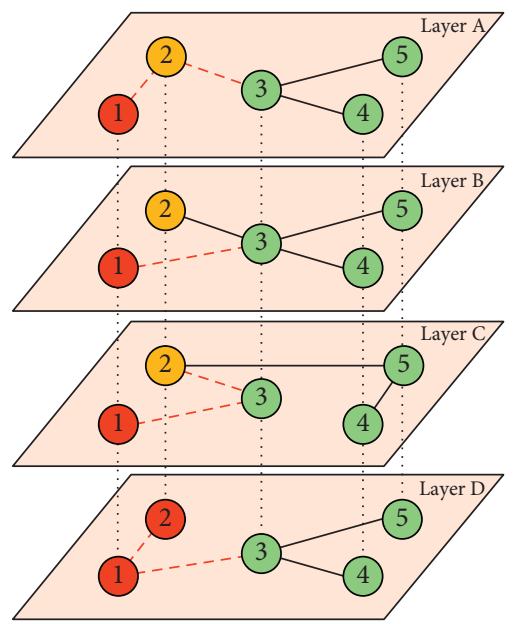

(b)

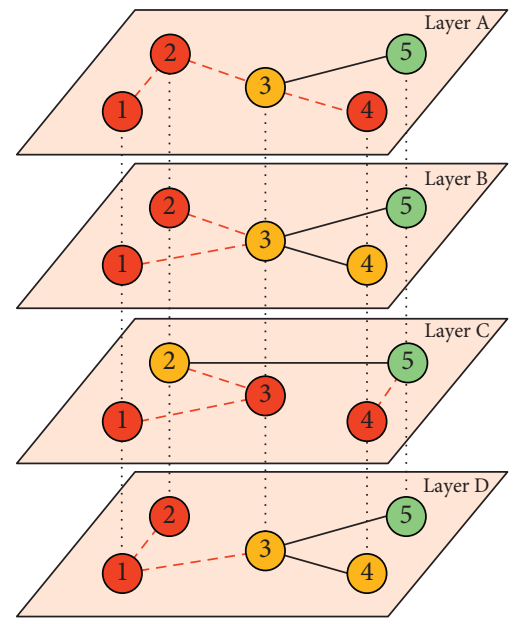

(e)

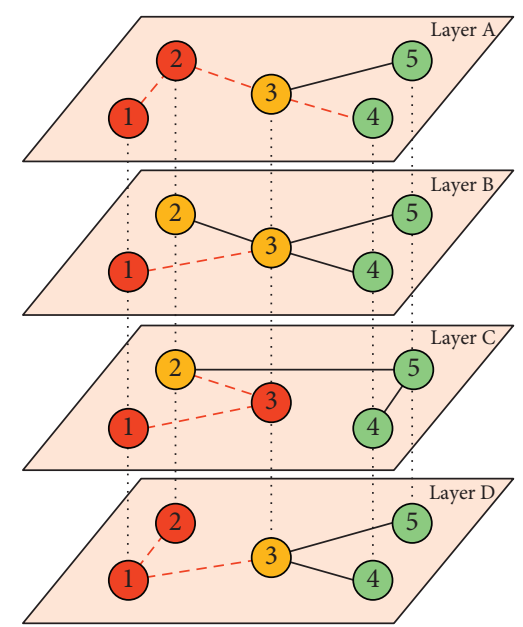

(c)

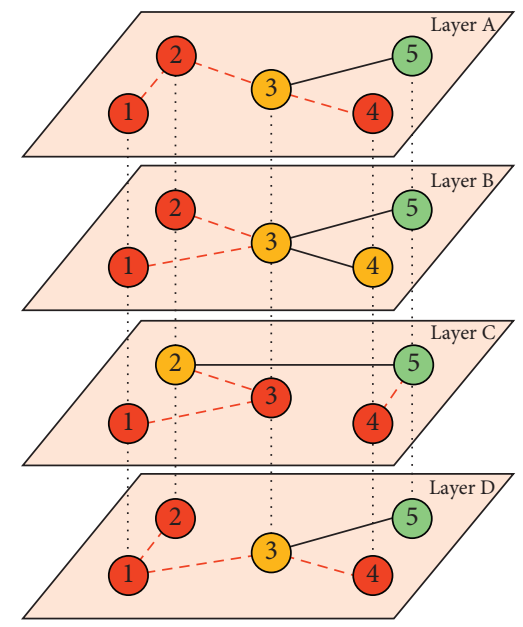

(f)

FIGURE 1: Schematic diagram of the cascading process in a four-layer network. The nodes in different layers with the same Arabic number are replica nodes, and they are connected by dotted lines. The funtional links are marked in solid lines and the failed links are marked in dashed lines. The functional nodes are marked in green, the failed nodes are marked in red, and the yellow nodes are still viable after being damaged. At stage (A), the replicas with the Arabic number 1 are removed from all layers. At stage (B), the node $(2, D)$ becomes isolated from the giant component in layer $D$ and fails, which leads to the link removal of its replicas in layers $A, B$, and $C$. At stage (C) the node (3, $C$ ) becomes isolated from the giant component in layer $C$ and fails, which leads to the link removal of its replicas in layers $A, B$, and $D$. Simultaneously, node $(2, A)$ becomes isolated and fails due to the removal of link 23 in layer $A$, which further leads to the link removal of its replicas in layers $B$ and $C$. At stage (D), the node $(4, A)$ becomes isolated from the giant component in layer $A$ and fails, which leads to the link removal of its replicas in layers $B, C$, and $D$. Simultaneously, node $(2, A)$ becomes isolated and fails due to the removal of link 23 in layer $B$. At stage $(E)$, the node $(4, C)$ becomes isolated from the giant component and fails, which leads to the link removal of its replicas in layers $B$ and $D$. At stage $(\mathrm{F})$, the node $(4, D)$ becomes isolated and fails, and the system reaches the final steady state. (a) Stage A. (b) Stage B. (c) Stage C. (d) Stage D. (e) Stage E. (f) Stage F.

node failures in its replicas. Schematic illustration of cascading process of a four-layer system is shown in Figure 1. In this paper, we use the relative size $S^{X}$ of the giant component in each layer of network $X$ to measure the robustness of the network.

\section{Theory}

We use the method of probability generation functions $[50,51]$ to obtain the theoretical solution of the model, and the generating function $G_{0}^{X}(x)=\sum_{k} p_{k}^{X} x^{k}$ is employed to generate the degree distribution $p_{k}^{X}$ of layer $X$. Similarly, the generating function $G_{1}^{X}(x)=\sum_{k} p_{k}^{X} k x^{k-1} /\langle k\rangle^{X}$ is used to generate the excess degree distribution of a node reached by following a random link, where $\langle k\rangle \equiv \sum_{k} p_{k}^{X} k$ represents the average degree of the network layer $X$. In particular, we define $R^{X}$ as the probability that a randomly chosen link in network $X$ leads to its giant component in the steady state of the system. For the case where the $M$ network layers in the system have a same degree distribution, $p_{k}^{X}=p_{k}$. Accordingly, $\quad G_{0}^{X}(x) \equiv G_{0}(x), \quad G_{1}^{X}(x) \equiv G_{1}(x), \quad R^{X} \equiv R, \quad$ and $\langle k\rangle^{X} \equiv\langle k\rangle$. Assuming that each network of $M$ network layers is tree-like, we aim to obtain the probability $S^{X}$ that a random node is in the giant component of layer $X$. Because 
each layer has the same degree distribution, we have $S^{A}=S^{B}=S^{C} \ldots \equiv S$. Following a randomly chosen link in the layer $X$, we arrive at a node $(x, X)$ of degree $k$ with $t$ failed replicas. Therefore, each link of node $(x, X)$ can be preserved with a probability $\alpha^{t}$. Considering that the degree $k$ follows the probability distribution $k p_{k} /\langle k\rangle$, the probability that a random link can lead to the giant component follows $\alpha^{t}\left[1-k p_{k} /\langle k\rangle\left(1-\alpha^{t} R\right)^{k-1}\right]$, which can be simplified as $\alpha^{t}\left[1-G_{1}\left(1-\alpha^{t} R\right)\right]$ in terms of the generating function $G_{1}(x)$. If the number $t$ of failed replicas for a given node follows a probability distribution $f(t)$, we can obtain the self-consistent equation for $R$ by summing over all possible $t$.

$$
R=p \sum_{t=0}^{M-1} \alpha^{t}\left[1-G_{1}\left(1-\alpha^{t} R\right)\right] f(t) \equiv h(R) .
$$

Similarly, we can obtain the probability $S$ that a random node is in the giant component:

$$
S=p \sum_{t=0}^{M-1}\left[1-G_{0}\left(1-\alpha^{t} R\right)\right] f(t) .
$$

The solution process of equations (1) and (2) utilizes the probability distribution function $f(t)$, which can be obtained by using the probability $R$. Considering that there are $t$ failed replicas for a random node $(x, X)$ in the layer $X$ at steady state, the viable probability of each replica is $1-G_{0}\left(1-\alpha^{t} R\right)$, and the remaining $M-t-1$ replicas are all viable with probability $\left[1-G_{0}\left(1-\alpha^{t} R\right)\right]^{M-t-1}$. Because the failures can propagate from layer to layer through the interdependencies among replica nodes, we assume that there are $s$ failed replicas caused by the link removal of other nodes in the corresponding network layers, and there are $t-s$ failed nodes induced by the $s$ failed replicas. The probability of $s$ failed replicas existing caused by isolation is $G_{0}^{s}(1-R)$. After that, the probability of $t-s$ additional replicas failing is $\left[G_{0}\left(1-\alpha^{s} R\right)-G_{0}(1-R)\right]^{t-s}$. Therefore, $f(t)$ satisfies

$$
\begin{aligned}
f(t)= & \left(\begin{array}{c}
M-1 \\
t
\end{array}\right)\left[1-G_{0}\left(1-\alpha^{t} R\right)\right]^{M-t-1} \sum_{s=0}^{t}\left(\begin{array}{l}
t \\
s
\end{array}\right) G_{0}^{s} \\
& \cdot(1-R)\left[G_{0}\left(1-\alpha^{s} R\right)-G_{0}(1-R)\right]^{t-s} .
\end{aligned}
$$

For a given degree distribution $p_{k}$, we can obtain the final size $S$ of the giant component in a certain layer by solving equations (1) and (2) simultaneously.

When $\alpha \longrightarrow 1$, the interdependence across network layers is weakest, and the system percolates in a secondorder manner as in single-layer networks [52, 53]. When $\alpha \longrightarrow 0$, the interdependence across network layers is the strongest, and the system percolates in a first-order manner [10]. Therefore, the manner of percolation transitions can be determined by the value of $\alpha$, and the critical value $\alpha_{c}$ is defined as the switch point of the percolation transition from a second-order to a first-order. For the second-order percolation transition, the probability $R$ tends to zero when $p$ is close to the second-order percolation point $p_{c}^{\mathrm{II}}$. We can use the Taylor expansion of equation (1) for $R \equiv \epsilon \longrightarrow 0$ and $p \longrightarrow p_{c}^{\mathrm{II}}$ :

$$
h(\epsilon)=h^{\prime}(0) \epsilon+\frac{1}{2} h^{\prime \prime}(0) \epsilon^{2}+O\left(\epsilon^{3}\right)=\epsilon .
$$

Ignoring the high-order terms of $\epsilon$, we have $h^{\prime}(0)=1$ when $p \longrightarrow p_{c}^{\mathrm{II}}$. We, thus, have the condition for the second-order percolation transitions

$$
p_{c}^{\mathrm{II}} \alpha^{2 M-2} G_{1}^{\prime}(1)=1,
$$

and the second-order percolation point

$$
p_{c}^{\mathrm{II}}=\frac{1}{\alpha^{2 M-2} G_{1}^{\prime}(1)} \text {. }
$$

When $\alpha=1$ or $M=1$, the second-order percolation transition point $p_{c}^{\mathrm{II}}=1 / G_{1}^{\prime}(1)$, which is coincident with the result for the ordinary percolation in a single-layer network [53]. In addition, we can also find the second-order percolation point $p_{c}^{\mathrm{II}}$ increases with the increase of $M$, which means the more network layers a system has, the more fragile it becomes.

At the first-order phase transition point $p_{c}^{\mathrm{I}}$, the probability $R$ jumps from $R_{c}$ to zero or a nontrivial value, and the curve of $y=h(R)-R$ is tangent with the straight line $y=0$ :

$$
\left.\frac{\mathrm{d} h(R)}{\mathrm{d} R}\right|_{R=R_{c}, p=p_{c}^{\mathrm{I}}}=1 \text {. }
$$

In this paper, we resort to the numerical method to solve equations (1) and (7) for the first-order percolation transition point $p_{c}^{\mathrm{I}}$.

When $\alpha=\alpha_{c}$, the conditions for the first- and secondorder percolation transitions are satisfied simultaneously, i.e., $p_{c}^{\mathrm{I}}=p_{c}^{\mathrm{II}}$ for $R_{c} \longrightarrow 0$ at the percolation transition point. At this time, equation (4) reduces to

$$
\frac{1}{2} h^{\prime \prime}(0) \epsilon^{2}+O\left(\epsilon^{3}\right)=0 \text {. }
$$

Therefore, we know that $h^{\prime \prime}(0)=0$ when $p \longrightarrow p_{c}$ and $\alpha=\alpha_{c}$. We can have

$$
\alpha^{3} G_{1}^{\prime \prime}(1)+2\left(\alpha^{2}-1\right)(M-1) G_{1}^{\prime}(1) G_{0}^{\prime}(1)=0 .
$$

By the solution of equation (9), we can obtain the switch point $\alpha_{c}$ of first-order and second-order percolation transitions. For multilayer random networks, the degree of nodes follows Poisson distribution, and equation (9) can be simplified as

$$
\alpha_{c}^{3}+(2 M-2) \alpha_{c}^{2}-(2 M-2)=0 .
$$

Therefore the value of $\alpha_{c}$ is only relevant to $M$. For scalefree networks, equation (9) can be written as

$$
\frac{\alpha_{c}^{3}\langle k(k-1)(k-2)\rangle}{\langle k\rangle\langle k(k-1)\rangle}+(2 M-2) \alpha_{c}^{2}-(2 M-2)=0,
$$

where the brackets $\langle\cdot\rangle$ denote the average over the degree distribution. Therefore, $\alpha_{c}$ depends not only on $M$ but also on the degree distribution $p_{k}$ for multilayer scale-free networks. 


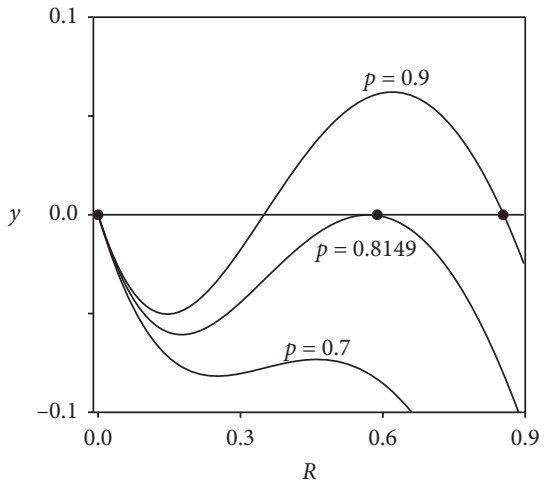

(a)

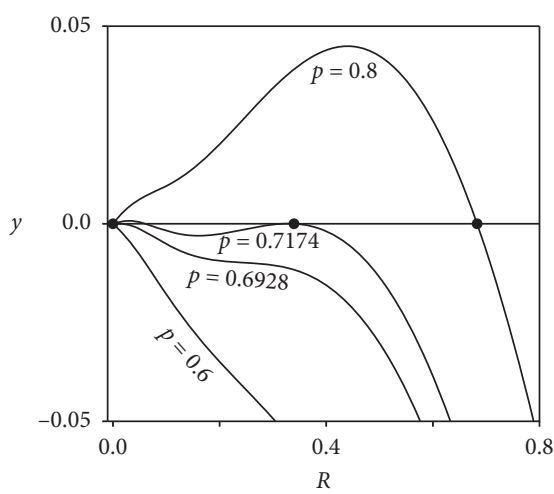

(b)

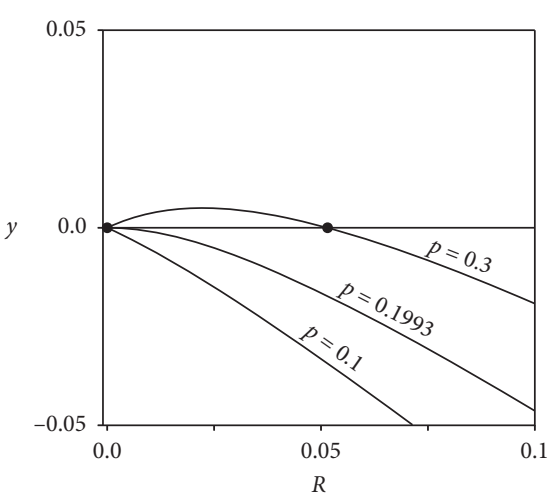

(c)

Figure 2: The graphical solutions of equation (1) for different values of $p$ and $\alpha$, as marked by the black dots. (a) The result for $\alpha=0.5$, (b) the result for $\alpha=0.65$, and (c) the result for $\alpha=0.9$. For each panel, the degree distribution of networks follows a truncated power-law distribution $p_{k} \sim k^{-\gamma}\left(k_{\min } \leq k \leq k_{\max }\right)$, where $k_{\min }=2, k_{\max }=141$, and $\gamma=2.3$.

Figure 2 shows the function curves $y=h(R)-R$ for different values of $p$, from which we can validate the existence of first- and second-order percolation transitions. Figure 2(a) shows the graphical solutions of equation (1) for $\alpha<\alpha_{c}$, from which we can find that the solution of $R$ is given by the tangent point when $p=p_{c}^{\mathrm{I}}$, indicating a discontinuous percolation transition. From Figure $2(\mathrm{c})$, we can find that the nontrivial solution of $R$ emerges at the point $p=p_{c}^{\text {II }}$, at which the function curve $y=h(R)-R$ is tangent with the $R$ axis at $R=0$, indicating a continuous percolation transition. Interestingly, we also found that the system undergoes double phase transitions for some moderate values of $\alpha$ if the degree distribution $p_{k}$ of the systems is scale-free, as shown in Figure 2(b). In this case, the system first percolates in a second-order manner and then experiences a first-order phase transition with increased $p$, and conditions (5) and (7) should be satisfied at the phase transition points $p_{c}^{\text {II }}$ and $p_{c}^{\mathrm{I}}$ successively. If condition (5) cannot be satisfied, the double phase transition reduces to a single first-order percolation transition, and if condition (7) cannot be satisfied, the double phase transition reduces to a single secondorder percolation transition. Using these conditions, we can locate the boundary between double phase transitions and single first-order phase transitions and the boundary between double phase transitions and single second-order percolation transitions.

\section{Results}

4.1. Synthetic Network. In this paper, we take two special multilayer networks with $M=3$ and $M=4$ layers as examples to illustrate the characteristics of percolation dynamics. Figures 3(a) and 3(b) show the size $S$ of the giant component as functions of $p$ for different values of $\alpha$ in three-layer random networks and four-layer random networks with $\langle k\rangle=4$, respectively. For both three-layer and four-layer random networks, we find that the system can percolate in a discontinuous manner for $\alpha=0.8$ or in a continuous manner for a larger value of $\alpha=0.95$. In addition, the percolation transition point of three-layer networks is less than that of four-layer networks, which means that three-layer networks are always more robust than four-layer networks. Simultaneously, we find that the critical point $\alpha_{c}$ separating the types of percolation transitions depends on the number of layers in the system. Similar results can also be found for three-layer random networks and four-layer random networks with $\langle k\rangle=5$. In Figure 3, the theoretical predictions for the size $S$ of the giant component as functions of $p$ and percolation transition points $p_{c}^{\mathrm{I}}\left(p_{c}^{\mathrm{II}}\right)$ are also provided, from which we can find that they agree with the simulation results very well.

The results for multilayer scale-free networks are also provided in Figure 4. For each network layer of the system, the degree of the nodes follows a truncated power-law distribution with an average $\langle k\rangle$, and the degree distribution is $p_{k} \sim k^{-\gamma}\left(k_{\min } \leq k \leq k_{\max }\right)$, where $k_{\min }$ and $k_{\max }$ are the minimum and maximum of the degree, respectively, and $\gamma$ denotes the power law exponent. Similarly, we can also find that the system can percolate in a discontinuous manner for a small value of $\alpha$ or in a continuous manner for a large value of $\alpha$, and the three-layer networks are more robust than fourlayer networks for both $\langle k\rangle=4$ and $\langle k\rangle=5$. Interestingly, we can also find that the four-layer networks can undergo a double phase transition for $\alpha=0.65$. Specifically, the system first percolates as a continuous phase transition and then undergoes a discontinuous phase transition with increasing $p$. With increasing $\alpha$, the discontinuous phase transition disappears, and the system reduces to a single continuous phase transition. With decreasing $\alpha$, the continuous phase transition disappears, and the system reduces to a single discontinuous phase transition.

Figures 5(a) and 5(b) show the percolation transition points $p_{c}$ as functions of $\alpha$ for three- and four-layer random networks with different average degrees, respectively. For both three- and four-layer random networks, the manners of percolation transition are classified as discontinuous and continuous by a critical value of $\alpha_{c}$, and the critical value of $\alpha_{c}$ only depends on the number of network layers $M$ in a system, which coincides the theoretical result provided by 


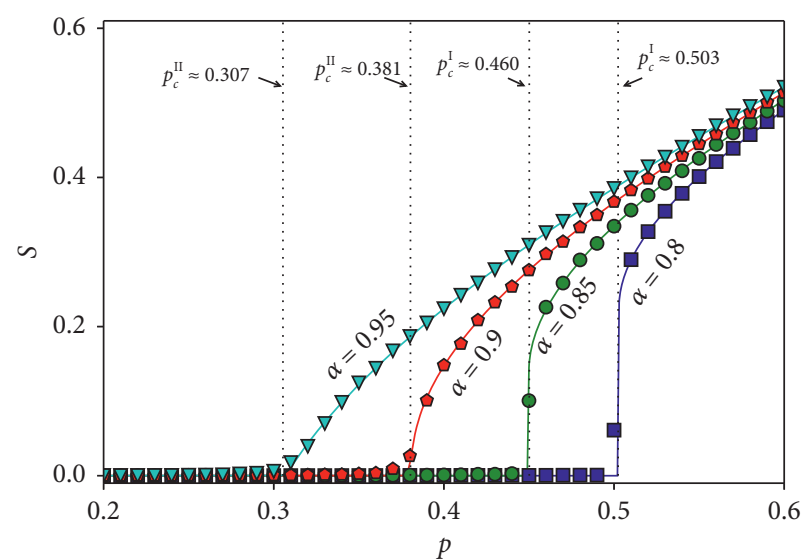

(a)

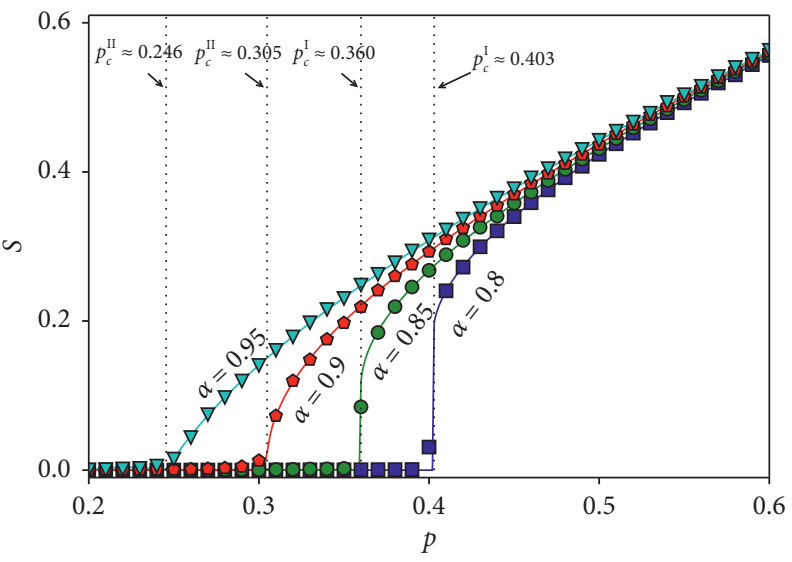

(c)

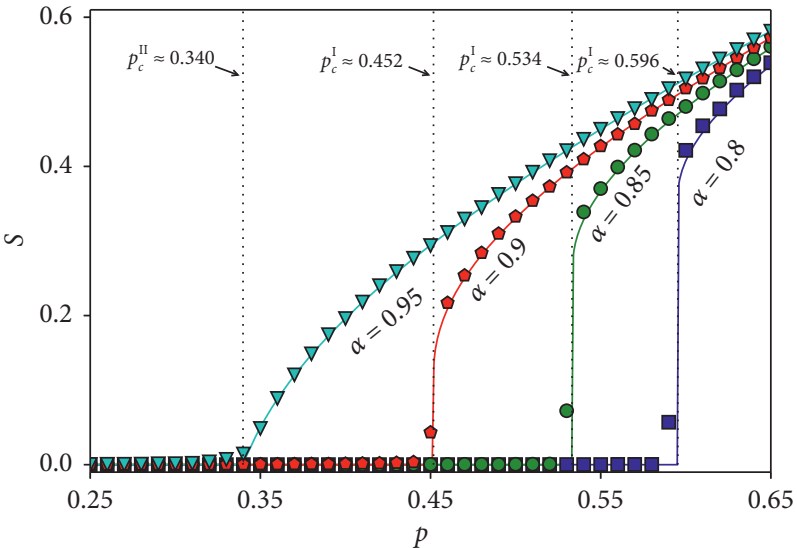

(b)

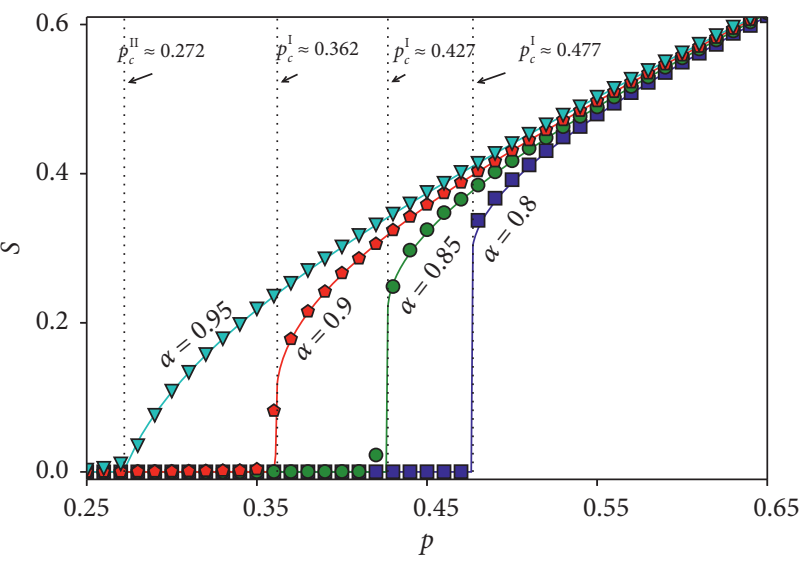

(d)

FIGURE 3: Simulation results for percolation transitions on three-layer and four-layer random networks. (a), (b) The results for three-layer and four-layer random networks with $\langle k\rangle=4$, respectively. (c), (d) The results for three-layer random networks and four-layer random networks with $\langle k\rangle=5$, respectively. The results were obtained by averaging over 100 independent realizations, and the network size was $N=10^{5}$. The solid lines behind the symbols denote the theoretical predictions that were obtained by equations (1) and (2). The vertical dashed lines denote the first- and second-order percolation transition points predicted by equations (5) and (7), respectively.

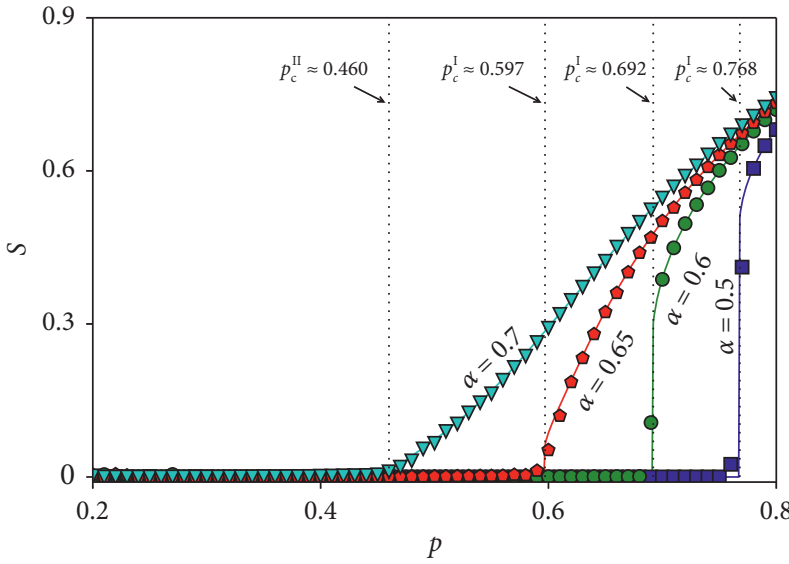

(a)

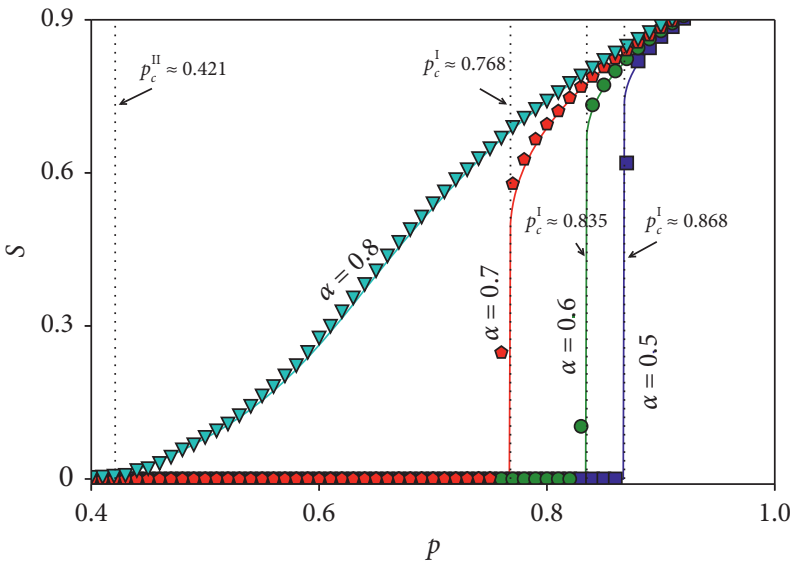

(b)

FIgURE 4: Continued. 


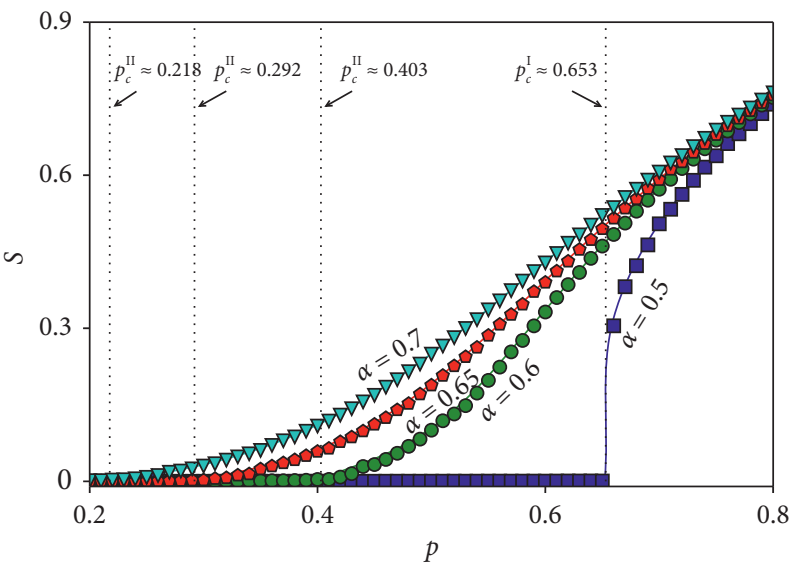

(c)

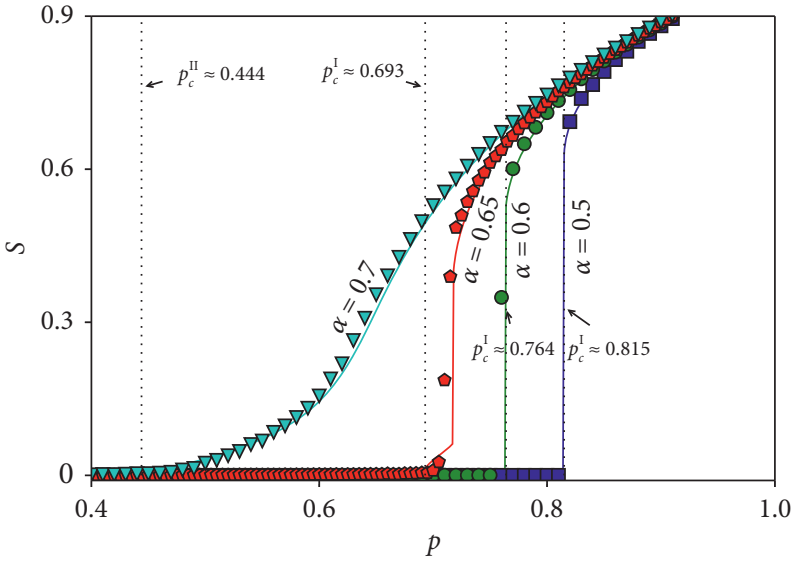

(d)

FIGURE 4: Simulation results for percolation transitions on three- and four-layer scale-free networks. (a), (b) The results for three- and fourlayer scale-free networks with $\langle k\rangle=4$, respectively. (c), (d) The results for three- and four-layer scale-free networks with $\langle k\rangle=5$, respectively. When the average degree $\langle k\rangle=4$, the parameter settings for the power-law distribution are $k_{\min }=2, k_{\max }=63$, and $\gamma=2.5$. When the average degree $\langle k\rangle=5$, the parameter settings are $k_{\min }=2, k_{\max }=141$, and $\gamma=2.3$. The simulation results were obtained by averaging over 100 independent realizations, and the network size is $N=10^{5}$. The solid lines behind the symbols denote the theoretical predictions that were obtained by equations (1) and (2). The vertical dashed lines denote the first- and second-order percolation transition points predicted by equations (5) and (7), respectively.

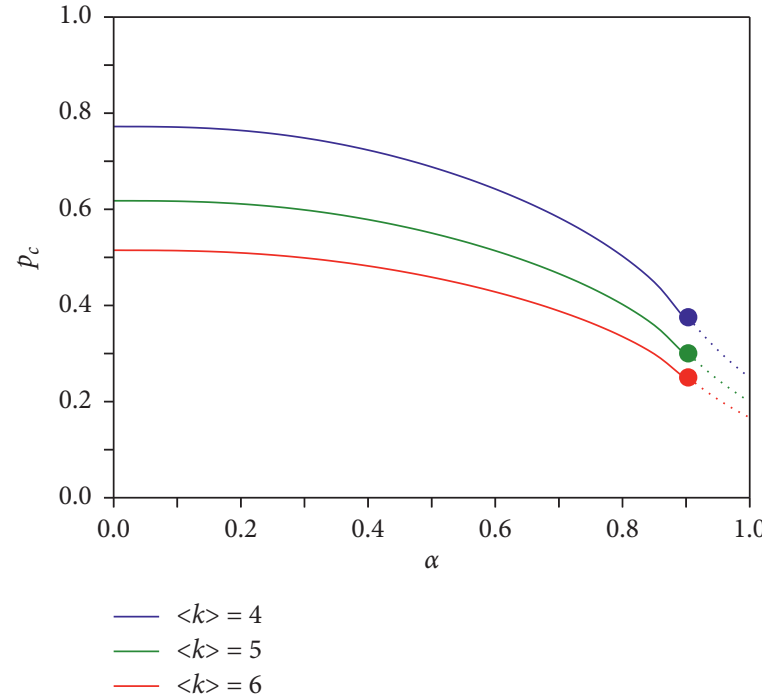

(a)

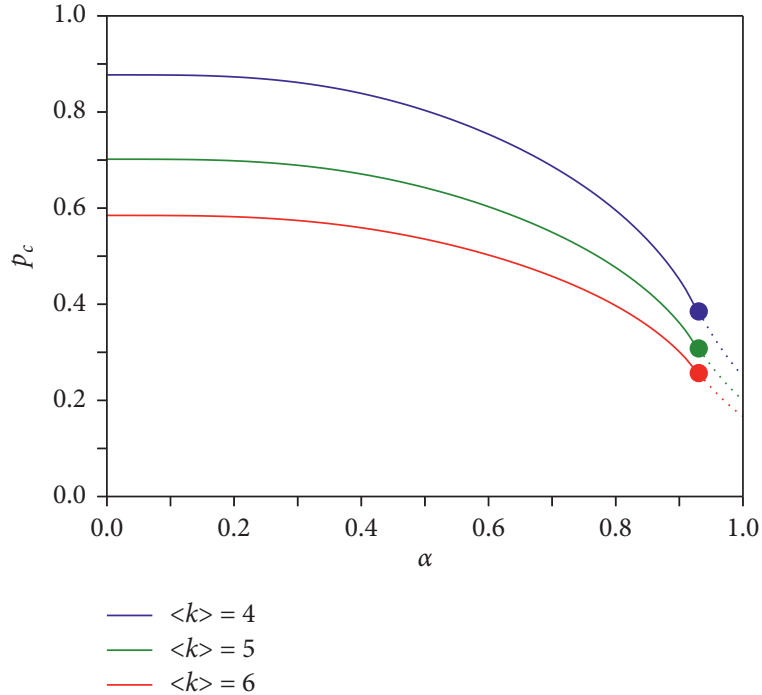

(b)

Figure 5: Continued. 


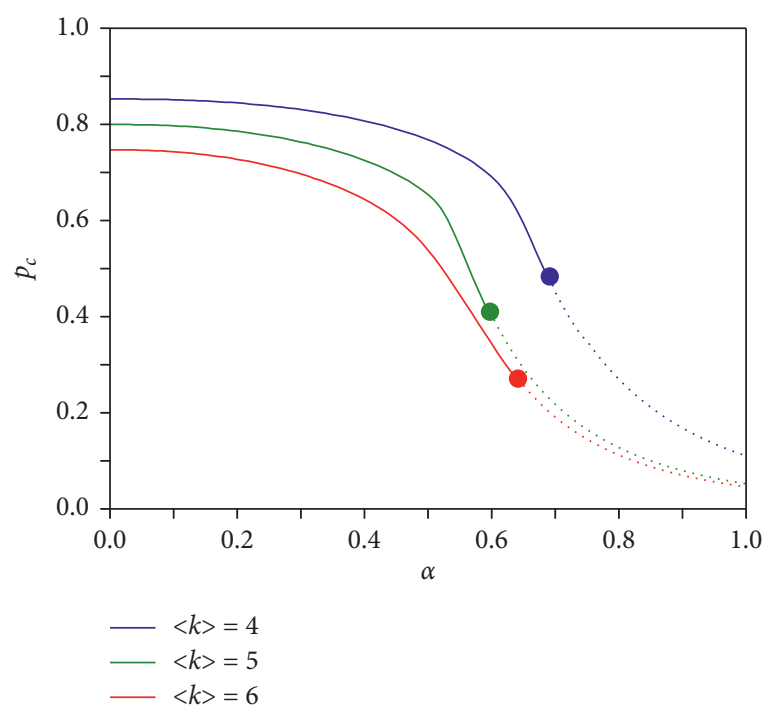

(c)

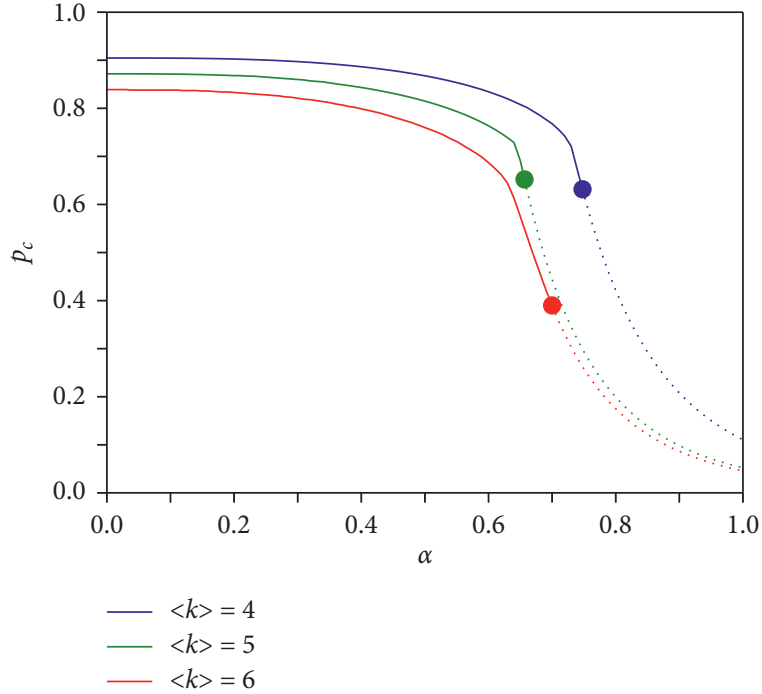

(d)

Figure 5: (Color online) (a) The percolation transition point $p_{c}\left(\left(p_{c}^{\mathrm{I}}\right)\right.$ or $\left.\left(p_{c}^{\mathrm{II}}\right)\right)$ versus $\alpha$ for three-layer random networks, where the average degree $\langle k\rangle$ is 4,5 , and 6 from top to bottom, respectively. (b) Corresponding results for four-layer random networks. (c) The corresponding results for three-layer scale-free networks of average degree $\langle k\rangle 4,5$, and 6 (corresponding to a power-law exponent of degree distribution $-2.6,-2.3$, and -2.1 from top to bottom, respectively) with minimum degree 2. (d) Corresponding results for four-layer scale-free networks.

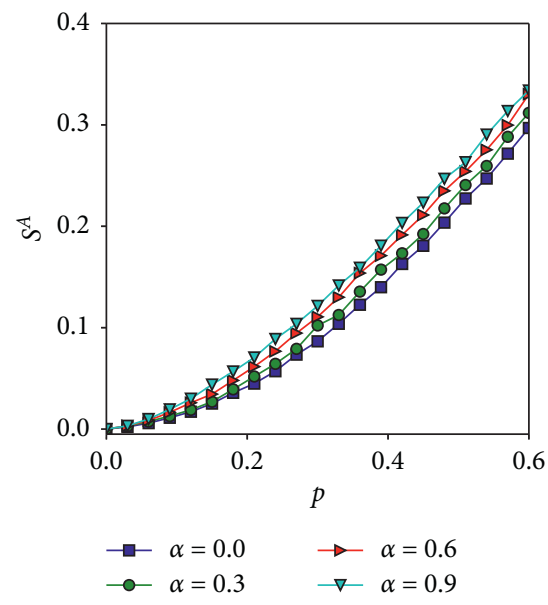

(a)

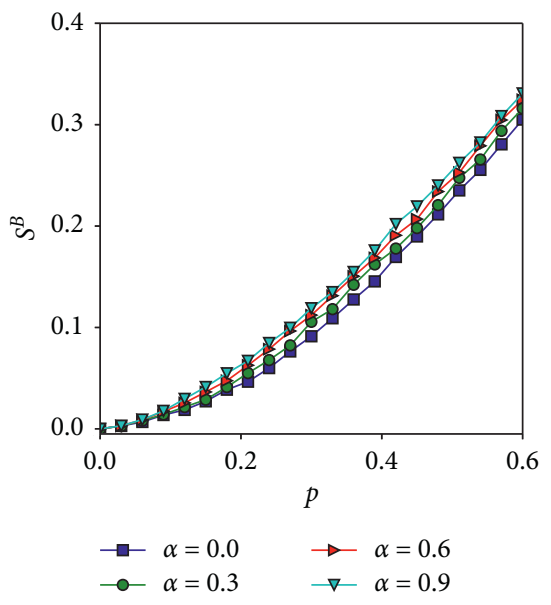

(b)

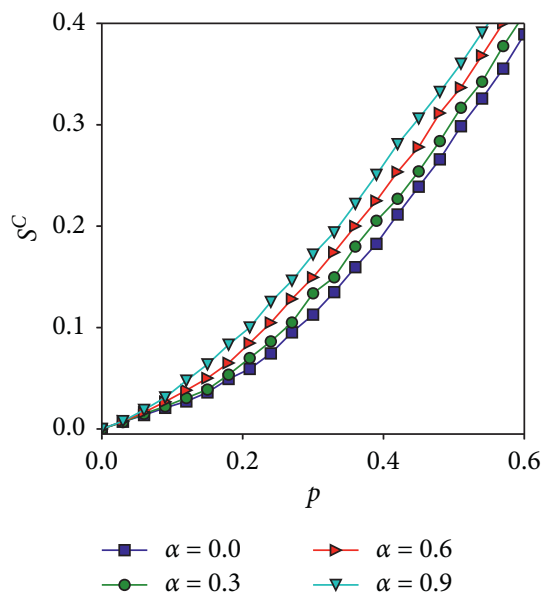

(c)

Figure 6: Percolation in multilayer empirical networks. The system consists of the three layers of networks A, B, and C which represent three major carriers: American Airlines (AA), Delta Air Lines (DL), and United Airlines (UA), respectively. (a-c) The sizes of the giant components of the network layers $A, B$, and $C$ as functions of $p$ for different values of $\alpha$, respectively. The data points are the result of averaging over 1000 statistical realizations. (a) AA. (b) DL. (c) UA.

equation (10). Figures 5(c) and 5(d) show the percolation transition points $p_{c}$ as functions of $\alpha$ for three- and fourlayer scale-free networks with different average degrees, respectively, from which we can also find that the manners of percolation transition are classified as discontinuous and continuous by a critical value of $\alpha_{c}$; however, the specific value of $\alpha_{c}$ depends on the parameter settings of the degree distributions. These results imply that the robustness of a multilayer network increases with the increase of the average degree of network layers and decreases with the increase of the number $M$ of network layers. For multilayer random networks, the collapse manner is irrelevant to the average degree of the networks, and abrupt breakdown cannot be avoided by the increase of the average degree. For multilayer scale-free networks, the collapse manner is relevant to the number $M$ of network layers and the parameters of degree distribution, i.g., minimum degree, maximum degree, and power law exponent. 
4.2. Empirical Networks. To address the percolation process in empirical multilayer networks, we consider a three-layer system constituting the three major carriers in the United States: Delta Air Lines (DL), American Airlines (AA), and United Airlines (UA). In each layer of a network, nodes represent airports, and links between two airports are connected in the layers if there is at least one flight operated by a given carrier. We construct the multilayer system using the dataset from OpenFlights (https://openflights.org/data. $\mathrm{html}$ ). For civil flights of the three major carriers, there are in total $N=310$ nodes (functioning airports). Some of the nodes do not appear as connected in all the layers, leading to a difference in the relative sizes of the giant components. Figures 6(a)-6(c)) show the sizes $S$ of the giant component as functions of $p$ for AA, DL, and UA, respectively. We can find that a large value of $\alpha$ always leads to a larger size $S$ of the giant component for three layers, which means that the robustness of the system can be improved greatly by restricting the interdependence across network layers with increasing $\alpha$.

\section{Conclusions and Discussion}

The interdependence of real multilayer networks is generally weak in layer-to-layer interactions, where the failure of one node usually does not result in failures of interdependent nodes across all network layers. In this paper, we have examined the percolation process and the robustness of a multilayer network when the interdependence of nodes across networks is weak. We reveal that the avalanche process of the whole system can be essentially decomposed into two microscopic cascading dynamics in terms of the propagation direction of failures: depth penetration and scope extension. Specifically, the former describes the propagation of failures across network layers and thus is regarded as "cross-layer cascading," while the latter describes the propagation of failures inside a network layer and, thus, is regarded as "inner-layer cascading." With the coaction of the two cascading dynamics, a multilayer network can disintegrate via first- or second-order percolation transitions in the case of initial failures, where the interdependence across network layers plays important roles in determining the percolation behaviors of the system. When the interdependence of network layers is weak, the failures of nodes can neither penetrate into deep network layers nor cause great destructiveness to their interdependent replicas, which inhibits the spread of failure and makes the system percolate via a second-order percolation transition. When the interdependence of network layers is strong, the failures of nodes can penetrate into deep network layers in a cascading manner and spread with a broad scope through various network layers, which, thus, makes the system collapse abruptly. These results prove that the process of "cross-layer cascading" dominates over the process of "inner-layer cascading" and plays a crucial role in determining the robustness of a multilayer system.

The present work essentially reveals the complexity of cascading failures, and previous works ignoring the weak interdependence may underestimate the complexity.
Specifically, the cascading dynamics that occur across network layers cannot be produced in a strong layer-to-layer interdependence of multilayer networks. Therefore, our method insights for the intervention of cascading failures in a multilayer network and evidences the idea that imposing restrictions on "cross-layer cascading" can restrain the spread of failures more effectively. Our work not only offers a new understanding of the cascading failure dynamics of multilayer networks but also implies that the strength of interdependence can be used for enhancing the robustness of multilayer structured infrastructure systems. That is to say, the penetration depth of cascading failures can be significantly reduced and thus the scale of cascading failures is also decreased by imposing restrictions on the strength of interdependence if the strength of interdependence is adjustable. For some other systems with fixed or nonadjustable interdependence across network layers, our finding is also meaningful for the robustness assessment. In this case, we can evaluate the robustness of such system by the parameters such as average degrees, number of network layers, strength of interdependence, and so on.

It has been found that overlapping links $[14,15]$ of real multilayer networks are able to relieve the destruction of cascading failures and improve robustness of the systems. Therefore, it is meaningful to study the percolation on multilayer networks with overlapping links in terms of tunable interdependency strength across networks in future works. Specifically, for a system with $M$ network layer, any two networks of it may have overlapping links and the degree of overlap may change with the varying of combination of network layers. We believe that the study of this issue will yield richer results.

\section{Data Availability}

The data of empirical networks used in this paper were downloaded from the website OpenFlights (https:// openflights.org/data.html), which contains basic data for multiple airports and flights worldwide, and we extract the data of civil flights of United States to built a three-layer network. The data of simulation used to support the findings of this study are available from the corresponding author upon request.

\section{Conflicts of Interest}

The authors declare that they have no conflicts of interest.

\section{Acknowledgments}

This work was supported by the National Natural Science Foundation of China under grant no. 61773148.

\section{References}

[1] D. F. Klosik, A. Grimbs, S. Bornholdt, and M.-T. Htt, “The interdependent network of gene regulation and metabolism is robust where it needs to be," Nature Communications, vol. 8, no. 1, p. 534, 2017. 
[2] M. Ouyang, "Review on modeling and simulation of interdependent critical infrastructure systems," Reliability Engineering \& System Safety, vol. 121, pp. 43-60, 2014.

[3] S. M. Rinaldi, J. P. Peerenboom, and T. K. Kelly, "Identifying, understanding, and analyzing critical infrastructure interdependencies," IEEE Control Systems, vol. 21, no. 6, pp. 11-25, 2001.

[4] F. Radicchi, "Percolation in real interdependent networks," Nature Physics, vol. 11, no. 7, pp. 597-602, 2015.

[5] J. Wu, J. Zhong, Z. Chen, and B. Chen, "Optimal coupling patterns in interconnected communication networks," IEEE Transactions on Circuits and Systems II: Express Briefs, vol. 65, no. 8, pp. 1109-1113, 2018.

[6] S. V. Buldyrev, R. Parshani, G. Paul, H. E. Stanley, and S. Havlin, "Catastrophic cascade of failures in interdependent networks," Nature, vol. 464, no. 7291, pp. 1025-1028, 2010.

[7] M. Kivela, A. Arenas, M. Barthelemy, J. P. Gleeson, Y. Moreno, and M. A. Porter, "Multilayer networks," Journal of Complex Networks, vol. 2, no. 3, pp. 203-271, 2014.

[8] J. Gao, S. V. Buldyrev, S. Havlin, and H. E. Stanley, "Robustness of a network of networks," Physical Review Letters, vol. 107, no. 19, Article ID 195701, 2011.

[9] J. Gao, S. V. Buldyrev, H. E. Stanley, and S. Havlin, "Networks formed from interdependent networks," Nature Physics, vol. 8, no. 1, pp. 40-48, 2012.

[10] G. J. Baxter, S. N. Dorogovtsev, A. V. Goltsev, and J. F. F. Mendes, "Avalanche collapse of interdependent networks," Physical Review Letters, vol. 109, no. 24, Article ID 248701, 2012.

[11] H. Kesten, Percolation Theory for Mathematicians, Birkhäuser Press, Boston, MA, USA, 1982.

[12] D. Stauffer and A. Aharony, Introduction to Percolation Theory, Tailor \& Francis Press, London, UK, 1992.

[13] S.-W. Son, G. Bizhani, C. Christensen, P. Grassberger, and M. Paczuski, "Percolation theory on interdependent networks based on epidemic spreading," EPL (Europhysics Letters), vol. 97, no. 1, p. 16006, 2012.

[14] D. Cellai, E. López, J. Zhou, J. P. Gleeson, and G. Bianconi, "Percolation in multiplex networks with overlap," Physical Review Letters, vol. 88, Article ID 052811, 2013.

[15] Y. Hu, D. Zhou, R. Zhang, Z. Han, C. Rozenblat, and S. Havlin, "Percolation of interdependent networks with intersimilarity," Physical Review E, vol. 88, no. 5, Article ID 052805, 2013.

[16] K.-K. Kleineberg, M. Boguñá, M. Ángeles Serrano, and F. Papadopoulos, "Hidden geometric correlations in real multiplexnetworks," Nature Physics, vol. 12, no. 11, pp. 1076-1081, 2016.

[17] K.-K. Kleineberg, L. Buzna, F. Papadopoulos, M. Boguñá, and M. A. Serrano, "Geometric correlations mitigate the extreme vulnerability of multiplex networks against targeted attacks," Physical Review Letters, vol. 118, no. 21, Article ID 218301, 2017.

[18] A. Faqeeh, S. Osat, and F. Radicchi, "Characterizing the analogy between hyperbolic embedding and community structure of complex networks," Physical Review Letters, vol. 121, no. 9, Article ID 098301, 2018.

[19] B. Min, S. D. Yi, K.-M. Lee, and K.-I. Goh, "Network robustness of multiplex networks with interlayer degree correlations," Physical Review E, vol. 89, no. 4, Article ID 042811, 2014.

[20] R. Parshani, C. Rozenblat, D. Ietri, C. Ducruet, and S. Havlin, "Inter-similarity between coupled networks," EPL (Europhysics Letters), vol. 92, no. 6, p. 68002, 2011.
[21] L. D. Ducruet, P. A. Macri, H. E. Stanley, and L. A. Braunstein, "Triple point in correlated interdependent networks," Physical Review E, vol. 88, no. 5, Article ID 050803, 2013.

[22] R.-R. Liu, C.-X. Jia, and Y.-C. Lai, "Asymmetry in interdependence makes a multilayer system more robust against cascading failures," Physical Review E, vol. 100, no. 5, Article ID 052306, 2019.

[23] R. Parshani, S. V. Buldyrev, and S. Havlin, "Interdependent networks: reducing the coupling strength leads to a change from a first to second order percolation transition," Physical Review Letters, vol. 105, no. 4, Article ID 048701, 2010.

[24] C. M. Schneider, N. Yazdani, N. A. M. Arajo, S. Havlin, and H. J. Herrmann, "Towards designing robust coupled networks," Scientific Reports, vol. 3, no. 1, p. 1969, 2013.

[25] L. D. Valdez, P. A. Macri, and L. A. Braunstein, "A triple point induced by targeted autonomization on interdependent scalefree networks," Journal of Physics A: Mathematical and Theoretical, vol. 47, no. 5, Article ID 055002, 2014.

[26] Y. Berezin, A. Bashan, M. M. Danziger, D. Li, and S. Havlin, "Localized attacks on spatially embedded networks with dependencies," Scientific Reports, vol. 5, no. 1, p. 8934, 2015.

[27] A. Bashan, Y. Berezin, S. V. Buldyrev, and S. Havlin, "The extreme vulnerability of interdependent spatially embedded networks," Nature Physics, vol. 9, no. 10, pp. 667-672, 2013.

[28] M. M. Danziger, A. Bashan, Y. Berezin, and S. Havlin, "Percolation and cascade dynamics of spatial networks with partial dependency," Journal of Complex Networks, vol. 2, no. 4, pp. 460-474, 2014.

[29] L. M. Shekhtman, Y. Berezin, M. M. Danziger, and S. Havlin, "Robustness of a network formed of spatially embedded networks," Physical Review E, vol. 90, no. 1, Article ID 012809, 2014.

[30] K. Gong, J.-J. Wu, Y. Liu, Q. Li, R.-R. Liu, and M. Tang, "The effective healing strategy against localized attacks on interdependent spatially embedded networks," Complexity, vol. 2019, Article ID 7912857, 10 pages, 2019.

[31] S. Shao, X. Huang, H. E. Stanley, and S. Havlin, "Robustness of a partially interdependent network formed of clustered networks," Physical Review E, vol. 89, Article ID 032812, 2014.

[32] X. Huang, S. Shao, H. Wang, S. V. Buldyrev, H. Eugene Stanley, and S. Havlin, "The robustness of interdependent clustered networks," EPL (Europhysics Letters), vol. 101, no. 1, p. 18002, 2013.

[33] T. Emmerich, A. Bunde, and S. Havlin, "Structural and functional properties of spatially embedded scale-free networks," Physical Review E, vol. 89, no. 6, Article ID 062806, 2014.

[34] X. Yuan, S. Shao, H. E. Stanley, and S. Havlin, "How breadth of degree distribution influences network robustness: comparing localized and random attacks," Physical Review E, vol. 92, no. 3, Article ID 032122, 2015.

[35] X. Yuan, Y. Hu, H. E. Stanley, and S. Havlin, "Eradicating catastrophic collapse in interdependent networks via reinforced nodes," Proceedings of the National Academy of Sciences, vol. 114, no. 13, pp. 3311-3315, 2017.

[36] L. B. Shaw and I. B. Schwartz, "Enhanced vaccine control of epidemics in adaptive networks," Physical Review E, vol. 81, no. 4, Article ID 046120, 2010.

[37] D. Zhao, L. Wang, S. Li, Z. Wang, L. Wang, and B. Gao, "Immunization of epidemics in multiplex networks," PLoS One, vol. 9, no. 11, Article ID e112018, 2014.

[38] J. C. Nacher and T. Akutsu, "Structurally robust control of complex networks," Physical Review E, vol. 91, no. 1, Article ID 012826, 2015. 
[39] Z. Wang, D. Zhou, and Y. Hu, "Group percolation in interdependent networks," Physical Review E, vol. 97, no. 3, Article ID 032306, 2018.

[40] R.-R. Liu, C.-X. Jia, and Y.-C. Lai, "Remote control of cascading dynamics on complex multilayer networks," New Journal of Physics, vol. 21, Article ID 045002, 2019.

[41] A. Bashan and S. Havlin, "The combined effect of connectivity and dependency links on percolation of networks," Journal of Statistical Physics, vol. 145, no. 3, pp. 686-695, 2011.

[42] A. Bashan, R. Parshani, and S. Havlin, "Percolation in networks composed of connectivity and dependency links," Physical Review E, vol. 83, no. 5, Article ID 051127, 2011.

[43] M. Li, R.-R. Liu, C.-X. Jia, and B.-H. Wang, "Critical effects of overlapping of connectivity and dependence links on percolation of networks," New Journal of Physics, vol. 15, no. 9, Article ID 093013, 2013.

[44] N. Azimi-Tafreshi, J. Gómez-Gardeñes, and S. N. Dorogovtsev, "k-core percolation on multiplex networks," Physical Review E, vol. 90, no. 3, Article ID 032816, 2014.

[45] G. J. Baxter, S. N. Dorogovtsev, J. F. F. Mendes, and D. Cellai, "Weak percolation on multiplex networks," Physical Review E, vol. 89, no. 4, Article ID 042801, 2014.

[46] F. Radicchi and G. Bianconi, "Redundant interdependencies boost the robustness of multiplex networks," Physical Review X, vol. 7, no. 1, Article ID 011013, 2017.

[47] R.-R. Liu, D. A. Eisenberg, T. P. Seager, and Y.-C. Lai, "The "weak" interdependence of infrastructure systems produces mixed percolation transitions in multilayer networks," Scientific Reports, vol. 8, no. 1, p. 2111, 2018.

[48] R.-R. Liu, M. Li, and C.-X. Jia, "Cascading failures in coupled networks: the critical role of node-coupling strength across networks," Scientific Reports, vol. 6, p. 35352, 2016.

[49] M. Molloy and B. Reed, "A critical point for random graphs with a given degree sequence," Random Structures \& Algorithms, vol. 6, no. 2-3, pp. 161-180, 1995.

[50] S.-W. Son, G. Bizhani, C. Christensen, P. Grassberger, and M. Paczuski, "Percolation theory on interdependent networks based on epidemic spreading," EPL, vol. 97, p. 16006, 2011.

[51] L. Feng, C. P. Monterola, and Y. Hu, "The simplified selfconsistent probabilities method for percolation and its application to interdependent networks," New Journal of Physics, vol. 17, Article ID 063025, 2015.

[52] R. Cohen, K. Erez, D. ben-Avraham, and S. Havlin, "Resilience of the internet to random breakdowns," Physical Review Letters, vol. 85, no. 21, pp. 4626-4628, 2000.

[53] D. S. Callaway, M. E. J. Newman, S. H. Strogatz, and D. J. Watts, "Network robustness and fragility: percolation on random graphs," Physical Review Letters, vol. 85, no. 25, pp. 5468-5471, 2000. 